\title{
Breast cancer: news in diagnosis and treatment
}

\author{
Afonso Celso Pinto Nazário ${ }^{1}$, Gil Facina ${ }^{2}$, José Roberto Filassi ${ }^{3}$ \\ ${ }^{1}$ Associate Professor, Habilitation (BR: Livre Docência), Head of the Division of Mastology at Escola Paulista de Medicina, Universidade Federal de São Paulo (EPM-UNIFESP), São Paulo, SP, Brazil \\ ${ }^{2}$ Adjunct Professor, Habilitation (BR: Livre Docência), Division of Mastology at EPM-UNIFESP, São Paulo, SP, Brazil \\ ${ }^{3}$ Associate Professor, Habilitation (BR: Livre Docência), Head of the Mastology Sector, Division of Gynecology, Department of Obstetrics and Gynecology. Hospital das Clínicas, Faculdade de Medicina, \\ Universidade de São Paulo, São Paulo, SP, Brazil
}

\section{Study conducted at Discipline of Mastology of the Departament of Gynecology at Escola Paulista de Medicina, Universidade Federal de São Paulo (EPM-UNIFESP) and at Sector of Mastology of the Discipline of Gynecology at Faculdade de Medicina da Universidade de São Paulo (FMUSP), São Paulo, SP, Brazil \\ Article received: 10/14/2015 Accepted for publication: 10/19/2015 \\ *Correspondence: \\ Address: Rua Nova York, 609 - apt. 73-L Postal code: 04560001 \\ São Paulo, SP - Brazil nazarioafonso@hotmail.com \\ http://dx.doi.org/10.1590/1806-9282.61.06.543 Conflict of interest: none}

\section{INTRODUCTION}

Breast cancer is currently the most common malignancy among women in developed countries, except for nonmelanoma skin tumors. In the United States (US), 231,840 new cases were estimated in 2015, which corresponded to $29 \%$ of cases of female cancer in the country. Brazil follows this trend, with an estimate in 2014 of 57,120 new cases, which makes it the most common malignancy among Brazilian women. In terms of incidence, the US saw an increase, between 1980 and 1987, of 4\% per year probably due to the intensification of mammographic screening programs. Between 1987 and 2002, this figure continued to grow, but at a lower rate ( $0.3 \%$ annually). Between 2002 and 2003, breast cancer incidence fell by $7.5 \%$, probably due to a decline of $50 \%$ in the prescription of hormone therapy for postmenopausal women in that country, after the release of the Women's Health Initiative (WHI) study, remaining stable after 2007. In addition to a high incidence, breast cancer also has a high mortality rate. It is the second leading cause of death due to malignancy among American women, second only to lung/ bronchial cancer. In 2015, it was responsible for 40,920 deaths in the US. However, the mortality rate, which grew between the years 1975 and 1990 at an annual rate of $0.4 \%$, declined between 1990 and 2002, presenting an annual drop of $2.3 \%$, possibly due to early detection and better treatment. In Brazil, it is responsible for the largest num- ber of deaths caused by malignant cancers among women and, unfortunately, the mortality rate remains high and growing, because the disease is still diagnosed in advanced stages. When the diagnosis is delayed, treatment is mutilating and aggressive. Thus, every effort should be made in order to detect the disease as soon as possible.

Breast cancer is the result of failure in the normal capacity of cells to proliferate and differentiate, which is caused by various genetic changes, culminating in malignant transformation. Mammary carcinogenesis is an extremely complex process and can be didactically divided into three stages: initiation, promotion and progression. At initiation, a phenotypically normal cell becomes malignant, usually presenting an intermediate stage of cellular atypia. Genetic mechanisms are involved in this stage and can be inherited (familial breast cancer) or acquired throughout life (sporadic breast cancer).

The familial form accounts for only $10 \%$ of cases of breast cancer and the leading genetic alteration found is inactivation of tumor suppressor genes. The genetic disorder is present right from the beginning of life, transmitted through germ line cells (autosomal dominant inheritance) and, therefore, tends to occur at an earlier age, i.e., premenopausal and often bilaterally. The main genes involved are $B R C A 1$ and BRCA2. BRCA1 is located on chromosome 17 and the carriers of this mutation have a high susceptibility to breast (65\% up to throughout life) and ovary (39\%) 
cancer. BRCA2 is located on chromosome 13 and is associated with high susceptibility to breast (45\%) and ovarian (11\%) cancer, as well as male breast cancer $(\mathrm{RR}=15)$.

In most cases, however, the genetic changes are not inherited but acquired during life, being a sporadic breast cancer ( $70 \%$ of cases). This is defined as a cancer in which no case is observed in two entire generations of $1^{\text {st }}$ and $2^{\text {nd }}$ degrees. The genetic defect usually results from activation of one or more proto-oncogenes present in healthy cells. Most oncogenes encode growth factors and their receptors. Familial breast cancer accounts for $20 \%$ of cases and, although there is a history in $1^{\text {st }}$ or $2^{\text {nd }}$ degree relatives, the autosomal dominant pedigree is not characterized. Through the activation of a proto-oncogene or the inactivation of suppressor genes, the modified cell transmits the error to next generation cells and so on. The genetic error can be repaired or the cell is induced to undergo apoptosis. When this does not occur, the genetic error can be perpetuated; the neoplastic cell is stimulated to divide forming mutated cell clones, which characterizes the promotion stage.

Among the factors that promote breast cancer, the most important are those that maintain the lobe in constant process of cell division, which hinders the physiological repair processes. External (exogenous) or internal (endogenous) stimuli favor the multiplication of cells containing genetic changes. The mutated cell clones, however, do not have the capacity to invade adjacent tissues, being restricted to the basal membrane (carcinoma in situ). Sex steroids and growth factors are very important for promotion. Thus, the main driving factors for breast cancer are of reproductive nature, such as early menarche, late menopause, nulliparity or oligoparity, and late first pregnancy. The common denominator is the continued cyclical estrogen-progestogen stimulation (ovulation) without the restorative break of pregnancy and lactation; in fact, the sex steroids act synergistically to keep the breast lobules in constant proliferation.

The pre-clinical stage, that is, the time interval between the first mutated cell and the formation of a malignant nodule measuring $1.0 \mathrm{~cm}$ (containing $10^{9}$ cells) is relatively long, approximately 8 years. However, once this volume is reached, the nodule's size doubles every 100 days. From a clinical point of view, at initiation and the primary stages of promotion, diagnosis using the available screening methods is not possible. But in the later stages of neoplastic promotion, with precursor lesions (atypical hyperplasia and ductal carcinoma in situ) already established, early detection through mammography is possible. It is believed that, between the normal epithelium and the invading carcinoma, intermediate stages of typical and atypical hyperplasia and in situ carcinoma can be seen, although not necessarily.

In the stage of progression, the already consolidated carcinoma breaks the basal membrane, invades blood and lymphatic vessels, and is capable of reaching tissues and proliferating at distance (metastasis). Angiogenesis and proteolytic enzymes, such as cathepsin $\mathrm{D}$, have an important role at this stage. The carcinoma begins to form palpable nodules that can be detected on physical and selfexamination. Inexorably, the disease progresses, produces distant metastases, the main sites being the bones, lungs and pleura, liver and brain.

\section{Diagnosis}

The diagnosis of breast cancer these days, very often, is done in its subclinical form, through routine mammography or population screening programs. In the US, mortality fell by $30 \%$ in women over 50 years old and $19 \%$ between 40 and 49 years on the account of earlier diagnosis. The BI-RADS ${ }^{\mathrm{TM}}$ (Breast Imaging Reporting And Data System) was implemented as a screening method, and constitutes a report and terminology standardization system that ranks the abnormalities seen on imaging studies into categories, as recommended by the American College of Radiology (Table 1).

\begin{tabular}{lll} 
TABLE 1 & BI-RADS \\
Category & classification. & \\
\hline 0 & Definition & Risk of cancer (\%) \\
\hline I & Inconclusive result & $\begin{array}{l}\text { Additional imaging } \\
\text { Investigation required }\end{array}$ \\
\hline II & Negative findings & 0 \\
\hline III & Benign findings & 0 \\
\hline IV & Probably benign findings & $<2$ \\
\hline V & Suspicious findings & $3-94$ \\
\hline VI & Highly suspicious findings & $\geq 95$ \\
& Known biopsy-proven & 100 \\
\hline
\end{tabular}

Breast exam has moderate sensitivity and good specificity for the detection of cancer, and the predictive value varies according to age. Sensitivity is from 57 and $83 \%$ between 50 and 59 years, and $71 \%$ between 40 and 49 years. Specificity, in turn, is greater, 88 to $96 \%$ between 50 and 59 years and 71 to $84 \%$ among women aged 40 to 49 years. It is particularly useful in younger women, due to the limitation of mammography in this age group (dense breasts).

Mammography is the standard method for screening and diagnosis of breast cancer. Even in cases where the clinical picture is suggestive, a mammogram should be indi- 
cated in order to assess the extent of the tumor and to detect subclinical ipsilateral multicentric foci, as well as in the contralateral breast. Sensitivity is 46 to $80 \%$, while specificity reaches 82 to $99 \%$. Its predictive value depends on the size and location of the lesion, breast density, the quality of technical resources available and the examiner's ability. However, even using a technique of excellence, the false negative rate is 10 to $15 \%$, reaching $40 \%$ in patients with dense breasts. Therefore, the clinical abnormality on palpation should not be neglected if the mammogram is normal, and the investigation should continue with other semiotic methods such as ultrasound, needle aspiration with thin or thick needle, or even conventional open biopsy.

The main mammographic signs of breast cancer are: (a) spiculated nodule (Figure 1); (b) clustered pleomorphic calcifications (Figure 2) and (c) focal asymmetry area with architectural distortion of the breast parenchyma. These images are associated with breast cancer in $90 \%$ of cases.

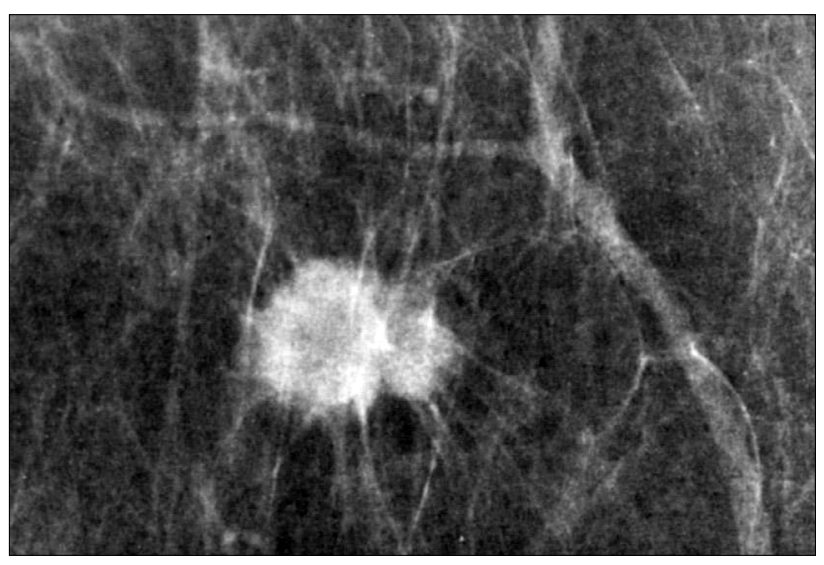

FIGURE 1 Spiculated nodule on mammography. BI-RADS ${ }^{\mathrm{TM}}$ V.

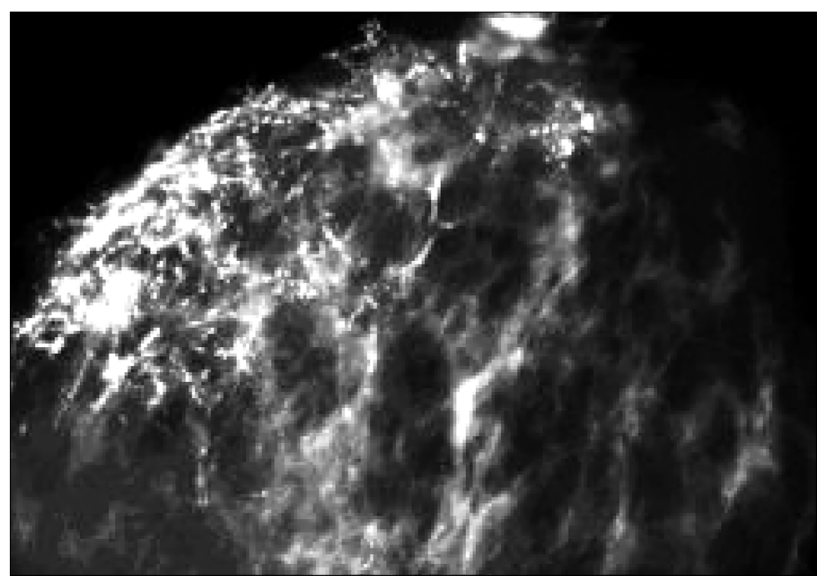

FIGURE 2 Grouped pleomorphic calcifications (appearance of Chinese letters). BI-RADS ${ }^{\mathrm{TM}} \mathrm{V}$.
Recently, digital mammography was introduced for diagnosis. Overall, the predictive value of this new method has not proved superior to analog; however, before menopause, in women under the age of 50 and with dense breasts, the diagnostic efficacy proved to be higher. In addition, the immediate analysis of the images on the workstation allows additional mammographic views to be promptly indicated, avoiding the need to re-examine the patient.

Although ultrasound is a non-ionizing and more comfortable method, it presents a lower predictive value compared to mammogram for diagnosis of breast cancer. It does not display less marked calcifications and, therefore, is not effective for screening as a single examination method. It should be indicated as an aid when mammogram does not establish a diagnosis, as in cases of asymmetry and dense breasts. The fat glands, on the other hand, hinder the transmission of the acoustic beam, limiting diagnosis.

From an ultrasound standpoint, breast cancer produces a solid irregular nodule, with imprecise margins and micro-lobulations or spicules (Figure 3 ). The internal echoes are heterogeneous, which produces a posterior acoustic shadow. The anteroposterior diameter of the malignant nodule is usually equal to or greater than the longitudinal diameter.

Magnetic resonance imaging is a new diagnostic method with increasingly important role in recent years. It is the imaging study with greater sensitivity for the detection of breast cancer, but must be requested with caution because its false positive rate is still not ideal, leading to unnecessary procedures. In addition, its cost is still high and its real impact in reducing mortality from breast can-

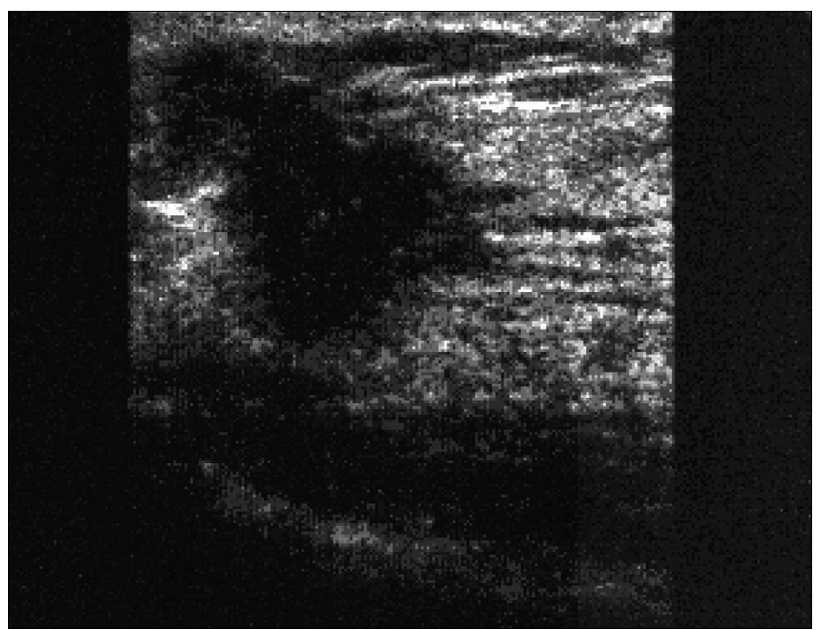

FIGURE 3 Sonographic appearance of breast cancer. Irregular spiculated nodule. 
cer is unknown. It is indicated when the extent of the suspected lesion cannot be determined by conventional methods, such as in dense breast tissue or when the diagnosis is lobular carcinoma; in women at high risk for developing breast cancer, such as the carriers of BRCA1 and BRCA2 gene mutations; to monitor the response to neoadjuvant chemotherapy; to assess the integrity of the prosthesis and in diagnoses of presumed occult breast carcinoma (i.e., presence of metastasis in the axilla without evidence of primary disease on clinical examination, mammogram and ultrasound).

Recently, tomosynthesis or 3D mammogram was introduced, with the promise to detect more lesions than digital mammography. However, its use as a routine did not prove to decrease mortality.

The American Cancer Society of Breast Diseases recommends that mammogram screening starts at the age of 40 years, being annual from this age. The Brazilian Ministry of Health, in turn, recommends that women at low risk for developing breast cancer undergo bi-annual mammograms, beginning at the age of 50 . In the case of high-risk women, the ministry advises that the examination should be carried out annually from the age of 35 , or 10 years before the age of diagnosis of the youngest affected relative. The Brazilian Society of Mastology suggests that mammogram is made annually between 40 and 49 years, and biannually thereafter. In 2015, the American Cancer Society began recommending that mammogram should be done annually between 45 and 54 years, and every two years thereafter. In São Paulo, the proportion of cases between 40 and 49 years $(21.3 \%)$ is very close to that found between 50 and 59 years $(27 \%)$. Thus, we recommend annual mammogram from the age of 40 years, ending when life expectancy is less than five years. Table 2 summarizes the various guidelines.

In cases of suggestive lesions, percutaneous biopsies are preferred. The first is biopsy with fine needle (FNAB), an outpatient method with low cost and relatively simple. The needle ( $25 / 6$ gange) makes the procedure comfortable and virtually risk-free. It can be done by palpation, but preferably ultrasound-guided, due to a higher predictive value. FNAB is contraindicated for investigation of suspected calcifications. Its disadvantages include the impossibility of evaluating invasion and histological grade. Moreover, it is very difficult to determine the histological type and the expression of hormone receptors. The false positive rate is very low (between 0 and $2 \%$ ). However, $5-20 \%$ of cases are false negatives, mainly due to improper technique for slide preparation. Thus, in cases of suspected image but negative, unsatisfactory or inconsis-
TABLE 2 Early detection and screening for breast cancer.

Brazilian Ministry of Health (2004)

\begin{tabular}{rr}
\hline Group without risk & $40-49$ years: Annual physical exam \\
\hline & $50-69$ years: Annual physical exam \\
\hline High-risk group & From the age of 35 years: Annual physical exam \\
\hline Annual mammograms
\end{tabular}

Brazilian Society of Mastology (2002)

40 - 49 years: Annual mammograms

50 - 69 years: Mammogram every two years

$\geq 70$ years: Depending on life expectancy

American Society of Breast Diseases (2006)

From the age of 40 years: Annual mammograms

American Cancer Society (2015)

45 - 54 years: Annual mammograms

After 55 years: Mammogram every two years (provided that life expectancy is greater than 10 years)

tent FNAB compared to the clinical-imaging diagnosis, investigation should continue.

Core needle biopsy (CNB) is done with a large needle and an automated device that propels and retracts the needle that cuts the tissue. It is indicated for suggestive nodules larger than $1.0 \mathrm{~cm}$ or extensive calcifications. Under those conditions, sensitivity is 48 to $100 \%$ and specificity, 91 to $100 \%$. On the other hand, it is contraindicated in case of deep image alterations (risk of pneumothorax and hemothorax), fibro-glandular distortion, in suggestive asymmetries, patients with hypomastia, or lesions adjacent to breast implant.

Advantages include the fact that it is an outpatient method, requiring local anesthesia. It allows evaluation of invasion, histological type and immunohistochemical expression, and the definition of a preoperative treatment planning. Cost (moderate), fragmentation of the material, and the mobilization of the breast tissue simulating invasion and/or embolization are the disadvantages. Anxious patients may find the procedure uncomfortable.

Mammotomy with core needle biopsy (CNB) is performed using a vacuum system with hollow cannula, which rotates at high speed, cutting through the tissue that is sucked out of the breast. It is also known as vacuum-assisted CNB. It is especially indicated for nodules smaller than $1.0 \mathrm{~cm}$, unpronounced suspected calcifications, fibro-glandular distortion and asymmetric densities. Sensitivity reaches $93 \%$ and specificity, $98 \%$.

As in the case of core biopsy, mammotomy has the advantage of being an outpatient method, using only local anesthetics. It makes it possible to evaluate invasion, 
histology and immunohistochemical markers. Moreover, the fragments removed are larger (in size and number), there is less mobilization of breast tissue, and the device is introduced once only, providing greater comfort to the patient, and also being more convenient for the physician. Finally, it allows pre-operative treatment planning. Disadvantages include discomfort that puncturing can produce in anxious patients and particularly the method's high cost.

Preoperative marking consists of preoperative location of suspected non palpable breast lesions. Can be made with a harpoon-shaped guide wire ("needling") (Figure 4) or by injection of a radioactive substance, a technique known as ROLL (radioguided occult lesion localization) (Figure 5). In both cases, marking can be performed using stereotactic mammogram or ultrasound.

When using stereotactic mammogram, the harpoon inserted before surgery should be placed $1.0 \mathrm{~cm}$ after the lesion, which allows it, even if the repair shifts, to continue to be close enough to the biopsy area. In ROLL, the scintigraphic emission is detected on the skin surface by gamma detector probe intraoperatively. It is more accurate and less invasive, but requires assistance of the nuclear medicine team and the cost is higher. In suspected calcifications,

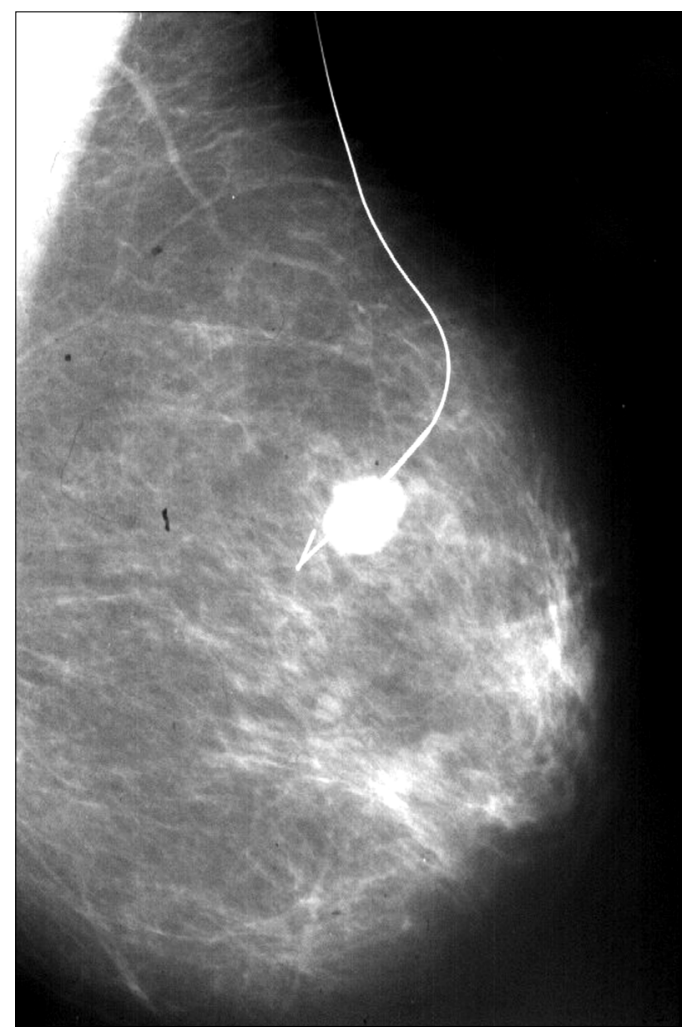

FIGURE 4 Preoperative marking with metallic harpoon - needling.

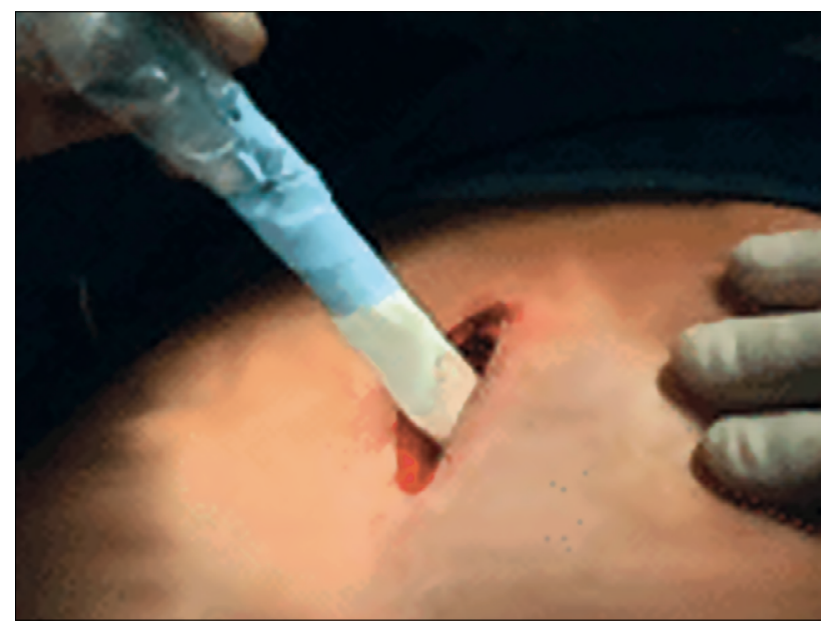

FIGURE 5 Intraoperative localization with gamma ray detection probe-ROLL.

mammogram of the surgical specimen is essential to confirm the removal of the previously marked lesion.

Preoperative marking has an advantage compared to methods of percutaneous biopsy, which is the fact that the material is abundant for pathological study, allowing better diagnostic definition and proper assessment of the resection margins. The main disadvantages are the need for hospitalization, anesthesia, a larger surgical scar, and the possibility of changes in subsequent images on mammogram and/or ultrasound. Furthermore, BI-RADS IV lesions are benign in $80 \%$ of cases on average, i.e., surgery is actually required in $20 \%$ of cases. The failure rate in removal ranges from 0.2 to $22 \%$, with an average of $4 \%$.

Once a diagnosis of breast cancer is made, it must be staged. The clinical stage of breast cancer is based on the TNM classification $(\mathrm{T}=$ tumor size; $\mathrm{N}=$ axillary lymph nodes; $M$ = distant metastases). Staging is both clinical (cTNM) and pathologic (pTNM).

\section{TREATMENT}

Halsted, in the late nineteenth century, proposed that breast cancer was a local disease, spreading it in an orderly and centrifugal manner to the axillary lymph nodes, which would act as an actual barrier to the spread of disease. Hence, the importance of resection of the breast and lymph nodes in the most radical way possible, to eradicate disease and improve survival. Highly radical surgical and radiotherapy prevailed in the first half of the twentieth century, but soon began to show disappointing results, because patients with small tumors treated in such an aggressive way would succumb, paradoxically, in relatively short times. 
Fisher, in the 1970s and 1980s, demonstrated through several clinical trials that the mechanistic theory of continuous spread of breast cancer was not appropriate. In fact, a tumor sized $1 \mathrm{~cm}^{3}$ has 1 billion cells and is capable of producing micro-metastases. This author proposed that breast cancer at a very early stage of development spreads via blood and can be regarded as a systemic disease from its beginning. He proposed that regional lymph nodes were not effective barriers and that their importance would lie only in the evaluation of the biological behavior and metastatic capacity of the cancer. In this way, lymphadenectomy would not influence survival, being important only as a prognostic factor. Fisher's theory led to the development, on scientific grounds, of several systemic treatments for breast cancer, including chemo and endocrine therapies, ultimately justifying the search for less aggressive and mutilating techniques to control locoregional disease.

The current consensus is that breast cancer should be considered a heterogeneous disease. Even though in most small tumors metastases are late, locoregional treatment being sufficient, there are cases in which metastatic spread occurs early and additional systemic treatment is needed. The selection of patients eligible for the appropriate systemic therapy is done by analyzing prognostic histopathological and immunohistochemical factors.

Surgical treatment of breast cancer in its initial stage (tumor size $\leq 3 \mathrm{~cm}$ ) consists in quadrantectomy and mammary segmental resection, i.e., removal of the quadrant or segment where the tumor is located (conservative treatment). For larger tumors, radical mastectomy sparing the pectoral muscles is usually recommended. Mastectomy is also indicated to treat the in situ multicentric form or invasive disease (Figure 6). Occasionally, when the breast is large and the relationship between tumor volume and breast volume is favorable, conservative surgery may be indicated for tumors between 3 and $5 \mathrm{~cm}$, associated with oncoplastic techniques.

In case of radical mastectomy, breast reconstruction may be performed, reducing the impact of breast amputation. Reconstruction can be immediate or delayed, and is usually done with transverse rectus abdominis (TRAM) flap reconstruction, with tissue expander and subsequent prosthesis insertion, or immediate prosthesis insertion, or latissimus dorsi myocutaneous flap. With TRAM, a mono or bipedicled flap of rectus abdominis muscle is moved up to the chest to rebuild the breast. The technique is complex, which increases the surgical time and use of anesthetics; also, the infusion of blood products is often required. However, this is the one that produces the best esthetic results in the long term. Using tissue expanders and implants, on the other hand, is faster and simpler; they are placed under the pectoralis major muscle and, in the case of expanders, gradually expanded in the postoperative period in order to increase skin elasticity and allow the later insertion of breast implants. They are indicated particularly for thin patients, who, therefore, have little subcutaneous abdominal tissue and would not benefit from the TRAM technique.

Axillary lymphadenectomy is performed when the axilla is medically compromised or when the tumor is large (greater than $5.0 \mathrm{~cm}$ ). Otherwise, the surgeon will remove the sentinel lymph node, which is the first lymph node to receive lymphatic drainage from the mammary gland.

The marking of the sentinel lymph node is basically done by two methods: dye or a radioactive tracer. In the first method, blue dye is injected into the peritumoral or periareolar region. After a few minutes, the dye is drained and marks the sentinel lymph node (Figure 7). In the second technique, radioisotope (usually technetium-99) is injected attached to a colloid, which binds to the sentinel lymph node. The injection is done hours before surgery and the success of the procedure is confirmed by preoperative lymphoscintigraphy. Radioactivity emitted by the marked lymph node is detected using a gamma detection probe during surgery (Figure 5). The technique has great predictive value, but requires specific surgical training, joint work of a nuclear medicine team, in addition to the cost being higher. With excision of the sentinel lymph node, routine extensive axillary dissection is avoided, as well as its unwanted side effects such as lymphoedema and neuropathy. However, the dissection of the sentinel lymph node is indicated only in early breast cancer when the axilla usually is not affected. Whenever intra-surgical frozen section examination is positive for malignancy, complete axillary lymphadenectomy is performed. Recently, a randomized clinical trial has shown that in tumors smaller than $5.0 \mathrm{~cm}$ treated conservatively followed by adjuvant systemic therapy and radiotherapy, when up to two sentinel lymph nodes are involved, resection of the sentinel nodes only had the same rates of local recurrence and overall survival compared to total axillary lymphadenectomy. Another study compared axillary lymphadenectomy and axillary radiotherapy in cases where sentinel node biopsy was positive, with no difference observed in terms of survival in both arms of the trial.

In quadrantectomy, or segmental excision of the breast, radiation therapy is formally indicated to prevent local recurrence in the rest of the gland. The breast is irradiated with a dose of $5000 \mathrm{cGy}$ (fractionated at 200 


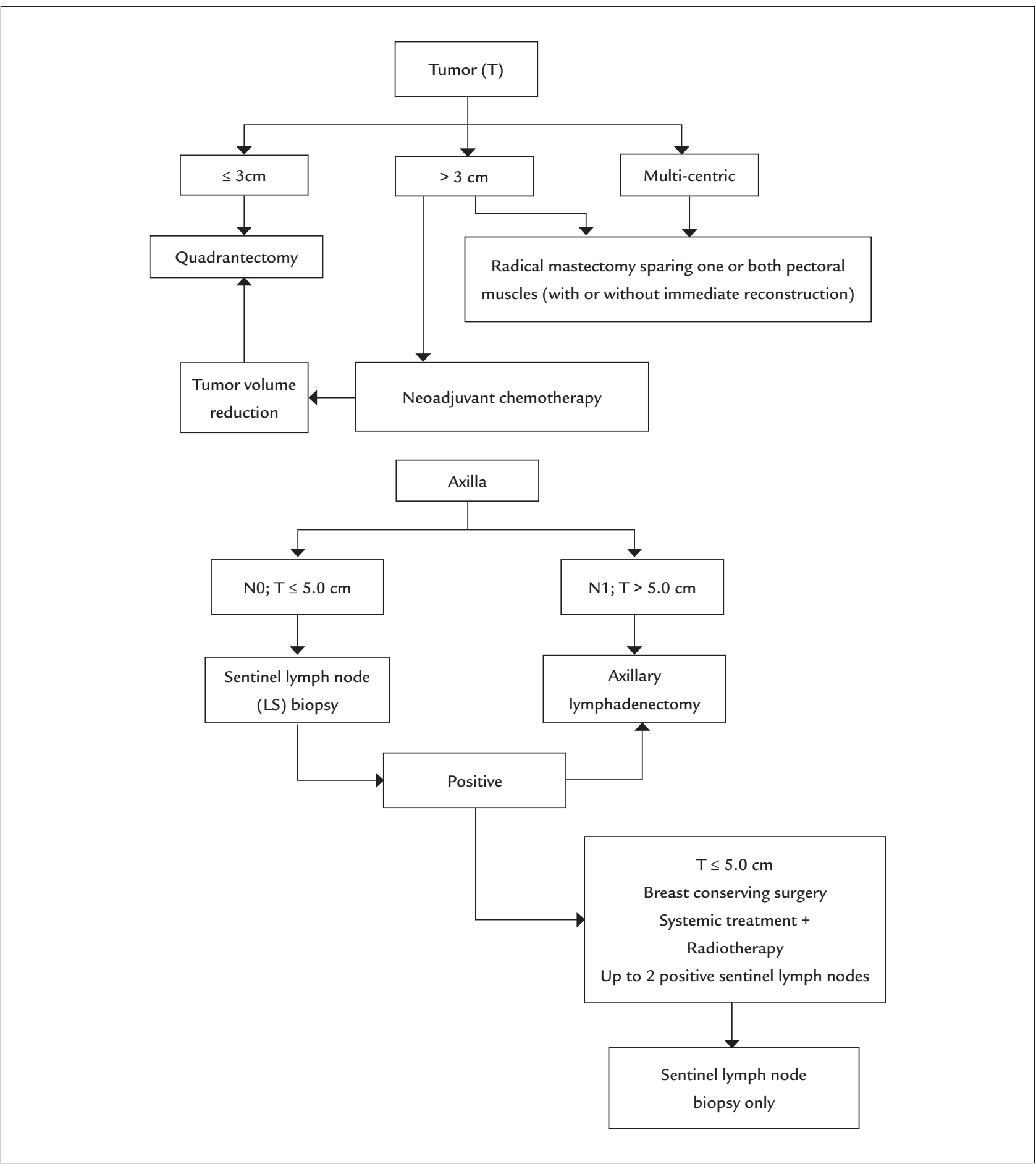

FIGURE 6 Surgical treatment flowchart of early breast cancer. 

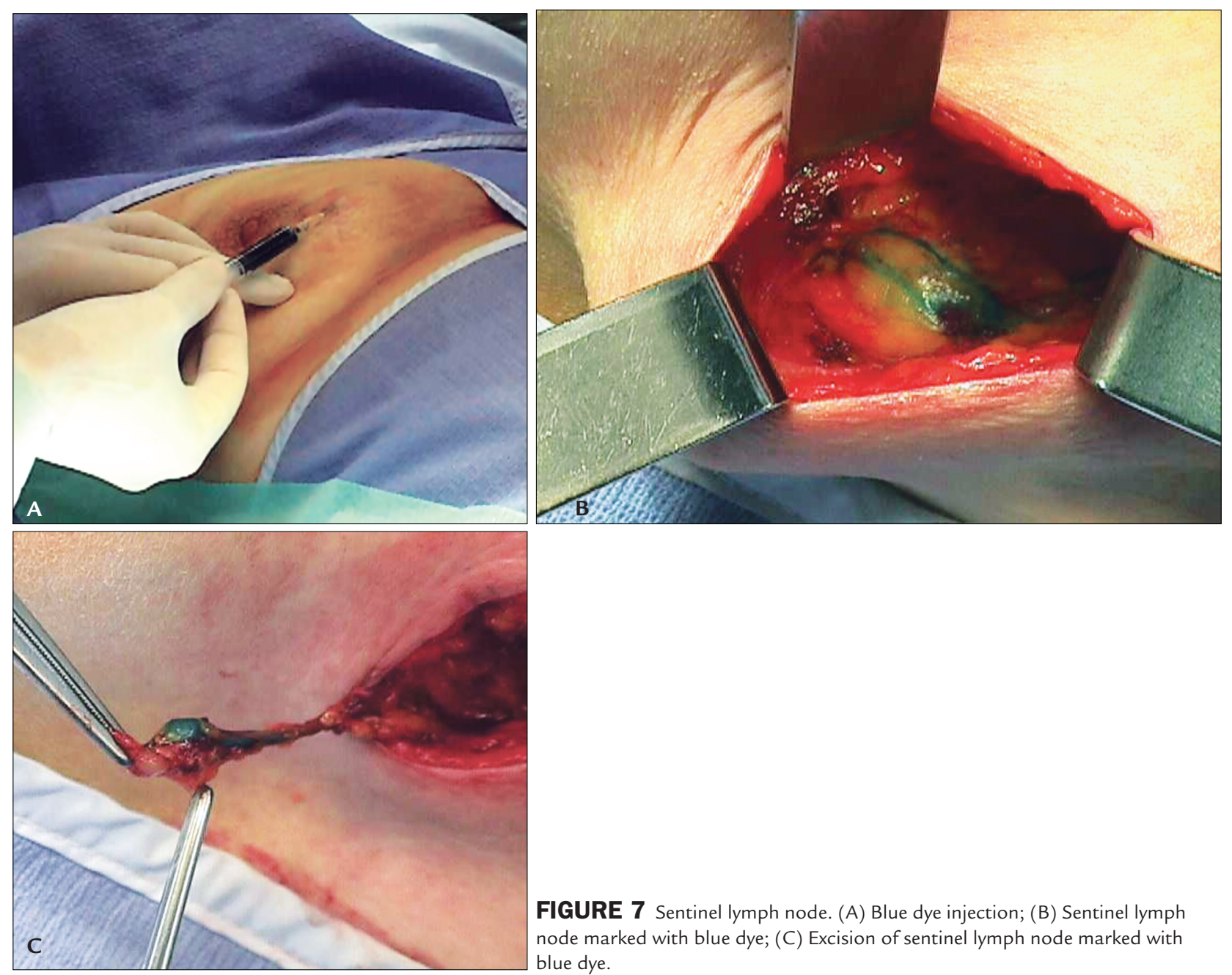

FIGURE 7 Sentinel lymph node. (A) Blue dye injection; (B) Sentinel lymph node marked with blue dye; (C) Excision of sentinel lymph node marked with blue dye.

cGy/day) and a booster of 1000 cGy (fractionated at 200 $c G y /$ day) in the tumor bed. When this treatment is not performed, local recurrence is very high, approximately $30 \%$ (versus $8 \%$ with radiation). Some protocols have been prepared to assess the effectiveness of the intraoperative radiation therapy, administered as a single dose. Although the preliminary results are promising, there is no long term follow-up data to determine the actual radiobiological effectiveness and safety. Randomized studies and meta-analyzes have shown that hypofractionated radiation, i.e. higher doses applied at shorter time intervals, is a safe and effective option for early breast cancer.

In tumors greater than $5 \mathrm{~cm}$, or when more than four lymph nodes are compromised, radiotherapy is also indicated after a mastectomy, with the same purpose, which is to prevent local recurrence. In this case, both the breast and lymphatic drainage chains (internal thoracic and supraclavicular) are irradiated. In recent years, even when axillary lymph node status is not high (one to three lymph nodes), there is a tendency to indicate the same adjuvant radiotherapy after mastectomy. Finally, radiation therapy may be indicated in bone metastases, particularly when there is pain or risk of pathological fracture, and in brain metastases.

The main prognostic factors for breast cancer include: tumor size, axillary lymph node status, histological type, histological grade, hormone receptors and c-erbB2.

Tumor size is directly related to prognosis and the axillary involvement. Thus, the 5 -year survival for cancers smaller than $2 \mathrm{~cm}$ is $75 \%$, and drops to $16 \%$ when the tumor measures more than $7 \mathrm{~cm}$. When the tumor measures less than $5 \mathrm{~cm}$, axillary involvement occurs in $3 \%$ of cases; $35 \%$ when the tumor is larger than $1.5 \mathrm{~cm}$. Axillary involvement plays an important prognostic role. When there is involvement of 1 to 3 lymph nodes, the 10 -year survival is between 31 and $54 \%$. When more than four lymph nodes contain metastases, survival drops to $13 \%$.

The most common histologic type of breast cancer is the ductal or invasive of no special type ( $90 \%$ of cases), followed by lobular (5-8\%). In a small percentage of cas- 
es, other histological types with better prognosis, such as tubular, mucinous or colloid, adenocystic and papillary, are identified. Variants that have a worse prognosis include ductal, undifferentiated, signet ring cells, inflammatory and metaplastic carcinoma.

The histological grade takes into account the tubular capacity for differentiation of the tumor, its nuclear grade, and mitotic counts. A grade is awarded for each parameter and the final score determines whether the tumor is well differentiated (grade I), moderately differentiated (grade II) or poorly differentiated (grade III). The five-year survival in histological grade I carcinomas is $81 \%$, and $34 \%$ in grade III.

Hormone receptor assessment has become routine to assist in estimating the prognosis and prediction of response to endocrine therapy. Estrogen and progesterone receptors are analyzed. Immunohistochemical evaluation also includes the expression of c-erbB2 (HER2/ $\mathrm{Neu})$. C-erbB2 is the growth factor receptor encoded by the HER 2 proto-oncogene and is part of a system of growth factor receptors overexpressed in breast cancer, namely HER-1 (EGFR ou erb B1), HER3 (erb B3) and HER4 (erb B4). C-erbB2 (HER2) can be measured by conventional immunohistochemical study or Herceptest, standardized tests for the evaluation of HER-2 protein. They are both presented as $0,1+, 2+$ and $3+$ scores. HER- 2 can also be detected using FISH technique, an in situ molecular hybridization method using DNA probes labeled with fluorescein. Although more specific, this is a more costly method and required fluorescein microscopy. Thus, breast carcinomas are divided from the molecular point of view in luminal (positive for estrogen and/or progesterone receptors), triple-negative (negative for estrogen and progesterone receptors, and HER2 negative) and HER2 positive. Luminal carcinomas have the best prognosis.

Chemotherapy is formally indicated in metastatic (palliative chemotherapy) and locally advanced (neoadjuvant or primary chemotherapy) disease. Neoadjuvant chemotherapy is also recommended to reduce the tumor volume and allow conservative surgery in cases that initially would be eligible for mastectomy.

In early disease, chemotherapy is employed when the tumor measures more than $2.0 \mathrm{~cm}$ (adjuvant chemotherapy), or when the axilla is compromised. In tumors between 1.0 and $2.0 \mathrm{~cm}$, there is no consensus on indication, but there is a tendency to perform it when: (a) the carcinoma is poorly differentiated; (b) hormone receptors and HER 2 are negative (triple-negative breast cancer); (c) when HER2 is overexpressed, and (d) the patient is under 40 years of age.
Recently, panels were set up to evaluate the expression of several genes involved in proliferation and to assist in deciding whether initial chemotherapy should be indicated for hormone receptor-positive carcinomas without axillary involvement, such as the oncotype and mammaprint. When the scores show high risk of recurrence, chemotherapy associated with endocrine therapy is indicated.

The most used chemotherapy drugs are cyclosporin, anthracyclines (adriamycin and epidoxorubicin) and taxanes (paclitaxel and docetaxel). When the expression of HER-2 is positive $(\mathrm{c}-\mathrm{erbB2} 3+$ or FISH + ), treatment with herceptin (trastuzumab) is recommended. Chemotherapy drugs are varied and many schemes are effective in treating breast cancer. In the initial disease, particularly when the axilla is not compromised, there is a tendency of using the $\mathrm{CMF}$ (consisting of cyclophosphamide at a dose of $500 \mathrm{mg} / \mathrm{m}^{2}$, methotrexate $70 \mathrm{mg} / \mathrm{m}^{2}$ and fluorouracil $500 \mathrm{mg} / \mathrm{m}^{2}$ ) and AC (cyclophosphamide $500 \mathrm{mg} / \mathrm{m}^{2}$ and epidoxorubicin $60 \mathrm{mg} / \mathrm{m}^{2}$ ) regimens. The drugs are administered intravenously, in a day, and repeated every 14 days for 4 cycles. In advanced breast cancer or in patients with compromised axilla, FAC, FEC or $\mathrm{AC}$ are preferred. In the latter case, after completion of the AC regimen, paclitaxel is added at a dose of $175 \mathrm{mg} / \mathrm{m}^{2}$, intravenously every 14 days, completing other 4 cycles. Some trials have demonstrated the efficacy of the combination of gemcitabine and cisplatin, but the results are still preliminary.

Trastuzumab is a humanized monoclonal anti-HER2 antibody and is indicated in patients with breast cancer overexpressing that oncogene. The combination of trastuzumab with taxanes improves survival of patients with metastatic breast cancer and several studies have demonstrated its effectiveness as well as adjuvant. It is administered every three weeks for one year.

Patients with hormone receptor-positive breast cancer should receive endocrine therapy. The answer is better when both receptors are positive. Treatment begins after the end of chemotherapy, if performed. The main form of endocrine therapy is tamoxifen at a dose of 20 mg per day orally during five years; recent randomized study demonstrated that the use of tamoxifen for 10 years was associated with a lower recurrence rate and longer survival, as compared with treatment for five years. Tamoxifen is a competitive antagonist of estrogen receptor in breast cancer. Its main contraindication is a personal history of deep vein thrombosis. In recent years, it has been shown that the use of aromatase inhibitors third generation after menopause produces more benefits to reduce the rate of recurrence compared to tamoxifen. Thus, aromatase inhibitors should be included at some point in endocrine therapy after menopause. 
After menopause, estrogen production occurs at the expense of the peripheral conversion of androgens to estrogens mediated by aromatase, which is abundant in fatty tissue. Thus, aromatase inhibitors present a more rational fundament, because they prevent the production of estrogen, while tamoxifen blocks estrogen, which has already been produced. Inhibitors may bind irreversibly (exemestane) or reversible (anastrozole and letrozole) to aromatase, and can be given in three manners: (a) sequentially for five years after the usual five years of tamoxifen therapy; (b) sequentially after two to three years of tamoxifen and, in this case, aromatase inhibitors are continued until the completion of five years of endocrine therapy; (c) single isolated form, for five years. The best scheme, which results in longer survival, has not yet been defined. The main side effects are hot flushes, arthralgia, myalgia and the negative effect on bone mass, increasing the risk of osteoporosis. Anastrozole, letrozole and exemestane are prescribed orally at doses of $1 \mathrm{mg}, 2.5 \mathrm{mg}$ and $25 \mathrm{mg}$, respectively.

Fulvestrant is an estrogen receptor antagonist, producing degradation and blocking transcription. It is free of agonist effects after binding to the receptor and it is used at a dose of $500 \mathrm{mg}$, once a month, intramuscularly. The drug is indicated for cases of relapse after treatment with tamoxifen or aromatase inhibitors.

\section{Resumo}

Câncer de mama: novidades no diagnóstico e no tratamento

Os autores discutem as principais novidades no diagnóstico e no tratamento do câncer de mama, particularmente no diagnóstico por imagem, no rastreamento e nas terapêuticas locorregional e sistêmica.

Palavras-chave: câncer de mama, diagnóstico, tratamento.

\section{REFERENCES}

1. American Cancer Society (ACS). [Viewed in november 28 $8^{\text {th }} 2015$ ]. Available from: http://www.cancer.org/cancer/breastcancer

2. American College of Radiology (ACR). Breast imaging reporting and data system (BI-RADSTM). 5th ed. Reston: American College of Radiology; 2013.

3. American Joint Committee on Cancer (AJCC). [Viewed in november $28^{\text {th }}$ 2015]. Available from: http://www.cancerstaging.org

4. Davies C, Pan H, Godwin J, Gray R, Arriagada R, Raina V et al. Long-term effects of continuing adjuvant tamoxifen to 10 years versus stopping at 5 years after diagnosis of oestrogen receptor-positive breast cancer: ATLAS, a randomised trial. Lancet. 2013; 381(9869):805-16.

5. Early Breast Cancer Trialists' Collaborative Group (EBCTCG), Darby S, McGale P, Correa C, Taylor C, Arriagada R et al. Effect of radiotherapy after breast-conserving surgery on 10-year recurrence and 15 -year breast cancer death: meta-analysis of individual patient data for 10,801 women in 17 randomised trials. Lancet. 2011; 378(9804):1707-16.
6. Fisher B, Redmond C, Fisher ER. The contribution of recent NSABP clinical trials of primary breast cancer therapy to an understanding of tumor biology - an overview of findings. Cancer. 1980; 46(4 Suppl):1009-25.

7. Fisher B, Costantino JP, Wickerham DL, Redmond CK, Kavanah M, Cronin WM et al. Tamoxifen for prevention of breast cancer: report of the National Surgical Adjuvant Breast and Bowel Project P-1 Study. J Natl Cancer Inst. 1998; 90(18):1371-88.

8. Giuliano AE, Dale PS, Turner RR, Morton DL, Evans SW, Krasne DL. Improved axillary staging of breast cancer with sentinel lymphadenectomy. Ann Surg. 1995; 222(3):394-9.

9. Giuliano AE, Hunt KK, Ballman KV, Beitsch PD, Whitworth PW, Blumencranz $\mathrm{PW}$ et al. Axillary dissection vs no axillary dissection in women with invasive breast cancer and sentinel node metastasis: a randomized clinical trial. JAMA. 2011; 305(6):569-75.

10. Glasziou PP, Woodward AJ, Mahon CM. Mammographic screening trials for women aged under 50. A quality assessment and meta-analysis. Med J Aust. 1995; 162(12):625-9.

11. Friedewald SM, Rafferty EA, Rose SL, Durand MA, Plecha DM, Greenberg JS et al. Breast cancer screening using tomosynthesis in combination with digital mammography. JAMA. 2014; 311(24):2499-507.

12. Fundação Oncocentro de São Paulo. Acesso ao TabNet - RHC Geral, 2015. Available from: http://www.fosp.saude.sp.gov.br/publicacoes/tabnet

13. Hayward OS. The history of oncology: I. Early oncology and the literature of discovery. Surgery. 1965;58:460-468.

14. INCA - Instituto Nacional de Câncer. Estimativa de incidência e mortalidade por câncer no Brasil para 2006. Available from: http:// www.inca.org.br

15. Johnson CH, van Heerden JA, Donohue JH, Martin JK Jr, Jackson IT, Ilstrup DM. Oncological aspects of immediate breast reconstruction following mastectomy for malignancy. Arch Surg. 1989; 124(7):819-23.

16. Kuhl C, Weigel S, Schrading S, Arand B, Bieling H, König R et al. Prospective multicenter cohort study to refine management recommendations for women at elevated familial risk of breast cancer: the EVA trial. J Clin Oncol. 2010; 28(9):1450-7.

17. Liberman L. Centennial dissertation. Percutaneous imaging-guided core breast biopsy: state of the art at the millennium. AJR Am J Roentgenol. 2000; 174(5):1191-9.

18. Lynch HT, Lynch J, Conway T, Watson P, Feunteun J, Lenoir G et al. Hereditary breast cancer and family cancer syndromes. World J Surg. 1994; 18(1):21-31.

19. Nazario AC, De Lima GR, Simões MJ, Novo NF. Cell kinetics of the human mammary lobule during the proliferative and secretory phase of the menstrual cycle. Bull Assoc Anat (Nancy). 1995; 79(244):23-7.

20. Nazario AC, Petti DA. Prevenção do câncer de mama. Jornal da Febrasgo. 2000; 7:5.

21. Nazário ACP (ed.), Elias S, Facina G, Araújo Neto JT (org.). Mastologia Condutas atuais. São Paulo: Manole; 2015.

22. Rossouw JE, Anderson GL, Prentice RL, LaCroix AZ, Kooperberg C, Stefanick $\mathrm{ML}$ et al. Risks and benefits of estrogen plus progestin in healthy postmenopausal women: principal results from the Women's Health Initiative randomized controlled trial. JAMA. 2002; 288(3):321-33.

23. SEER - National Cancer Institute Surveillance, Epidemiology and End Results. Database 2006. Available from: http:// www.cancernet.nci.nih.gov

24. Shapiro $S$. Evidence on screening for breast cancer from a randomized trial. Cancer. 1977; 39(6 Suppl):2772-82.

25. Singletary SE, Dhingra K, Yo DH. New strategies in locally advanced breast cancer. In: Pollock RE (ed). Advances in Surgical Oncology. Norwell: Kluwer Academic Publishers; 1996.

26. Slamon DJ, Leyland-Jones B, Shak S, Fuchs H, Paton V, Bajamonde A et al. Use of chemotherapy plus a monoclonal antibody against HER2 for metastatic breast cancer that overexpresses HER2. N Engl J Med. 2001; 344(11):783-92.

27. Tabár L, Vitak B, Chen TH, Yen AM, Cohen A, Tot T et al. Swedish two-county trial: impact of mammographic screening on breast cancer mortality during 3 decades. Radiology. 2011;260(3): 658-63.

28. Veronesi U, Saccozzi R, Del Vecchio M, Banfi A, Clemente C, De Lena M et al. Comparing radical mastectomy with quadrantectomy, axillary dissection, and radiotherapy in patients with small cancers of the breast. N Engl J Med. 1981; 305(1):6-11.

29. Veronesi U, Paganelli G, Galimberti V, Viale G, Zurrida S, Bedoni M et al. Sentinel-node biopsy to avoid axillary dissection in breast cancer with clinically negative lymph-nodes. Lancet. 1997; 349(9069):1864-7.

30. Yu YH, Liang C, Yuan XZ. Diagnostic value of vacuum-assisted breast biopsy for breast carcinoma: a meta-analysis and systematic review. Breast Cancer Res treat. 2010; 120(2):469-79. 